\title{
DEUTSCH-BOSNISCHER SPRACHKONTAKT AM BEISPIEL BOSNISCHER KULTURSPEZIFIKA IN MILENA PREINDLSBERGER-MRAZOVIĆS „DIE BOSNISCHE OSTBAHN““
}

\section{EINFÜHRUNG}

Auf dem Berliner Kongress im Sommer 1878 wurde Österreich-Ungarn das Mandat erteilt, Bosnien-Herzegowina zu okkupieren und nach dem dreijährigen Aufstand militärisch und politisch zu stabilisieren. Doch diese vormals von den Osmanen regierte Provinz wurde nicht nur militärisch besetzt: Obwohl Bosnien-Herzegowina völkerrechtlich immer noch Teil des Osmanischen Reiches war, wurde es in wenigen Jahren immer stärker an Österreich-Ungarn gebunden. Diese Eingliederung in die k. u. k. Monarchie erfolgte nicht nur politisch und militärisch, sondern auch gesellschaftlich und kulturell.

Tatsächlich war Bosnien-Herzegowina im 18. und 19. Jahrhundert durch häufige Aufstände und das weitgehend geschwächte Osmanische Reich in vielen Aspekten des Lebens ein gerütteltes und tief vernachlässigtes Land - für Österreich-Ungarn war die Okkupation im Jahre 1878 die Gelegenheit, durch die intensive politische, wirtschaftliche und auch kulturelle Einflussnahme in Bosnien-Herzegowina seine geostrategische Position auf dem Balkan weiter auszubauen. Die Eingliederung in die k. u. k. Zollunion, die Einführung der k. u. k. Wehrpflicht für bosnische Landesangehörige sowie die Reform der Glaubensgemeinschaften sind nur drei Beispiele dafür. In diesem Unterfangen bediente sich die Donaumonarchie durchaus kolonialer Ideen und Praktiken, die wir auch von anderen ,klassischen“ Kolonialmächten kennen. Die 40-jährige österreichisch-ungarische Verwaltung in Bosnien-Herzegowina wies laut einigen Forschern Elemente des sogenannten „Ersatzkolonialismus“ auf. Dieser manifestierte sich unter anderem durch die administrative Bevormundung, den Aufbau eines Wissensregimes, durch das Siedlerwesen sowie durch die Nutzung einer „Kulturmission“ als Werkzeug, um das Land weniger demokratisch zu regieren (vgl. Ruthner 2018: 36f.).

Um diese Machtpraktiken zu verstehen, wird hier das Beispiel der Migrationsfrage näher erörtert. Die österreichisch-ungarische Okkupationsmacht hatte wenig bis gar kein Vertrauen in den aus der osmanischen Zeit geerbten Verwaltungsapparat. Die neue k. u. k. Verwaltung bestand nämlich fast ausschließlich aus Beamten, die aus anderen Teilen der Donaumonarchie kamen - viele von ihnen aus dem südslawischen Raum. Mit ihnen kamen Bauern, Lehrer, Ingenieure, Geschäftsleute, Literaten und Forscher: Ihre Zahl stieg kontinuierlich. Nach der letzten österreichisch-ungarischen Volkszählung (1910) lebten in Bosnien-Herzegowina insgesamt 1.898.044 Personen, davon

nedad.memic@gmail.com 
waren 46.859 Personen österreichische und 61.151 Personen ungarische Staatsangehörige. Diese Zugezogenen wurden im bosnischen Volksmund abwertend „kuferaši“ (jene, die mit dem Koffer kamen) genannt - in diesem pejorativen Ausdruck spiegelt sich eine Abneigung der einheimischen Bevölkerung gegenüber der Okkupationsmacht und zahlreichen fremden Beamten und Kaufleuten wider, die ihr folgten. Der starke Zuzug lässt sich auch an den Zahlen festhalten: Hielten sich 189570.848 Personen aus der österreichischen und ungarischen Reichshälfte in Bosnien-Herzegowina auf, so ist ihre Zahl 1910 auf 114.591 angestiegen (Die Ergebnisse der Volkszählung 1912: 48f.).

\section{DEUTSCHE UND DEUTSCHSPRACHIGE IM K. U. K. BOSNIEN- HERZEGOWINA}

Die Volkszählung des Jahres 1910 verzeichnet u. a. 22.968 Personen in Bosnien-Herzegowina, deren Muttersprache Deutsch war (1,21\% der Gesamtbevölkerung). Die Präsenz der deutschsprachigen Bevölkerung in Bosnien-Herzegowina war nicht so zahlreich wie in manchen anderen österreichisch-ungarischen Regionen des Balkanraums wie Wojwodina, Slawonien oder auf slowenischem Gebiet (vgl. Krevs Birk 2019: 158-161). Trotzdem waren deutschsprachige Zuwandererinnen und Zuwanderer nach Bosnien-Herzegowina in ihrer sozialen Struktur heterogen: Zum einen wurden sie als Kolonisten in ländliche Gegenden Nordbosniens angesiedelt, zum anderen zogen sie auch in die größeren Städte des Landes. Die ländliche Zuwanderung deutscher Kolonisten nach Bosnien-Herzegowina begann mit der Gründung des Trappistenklosters Mariastern in der Nähe von Banja Luka durch den Pfarrer Franz Pfanner im Jahre 1869. Pfanner war zehn Jahre später der Initiator einer Siedlungsmigration nach BosnienHerzegowina, in dem er in deutschen katholischen Zeitungen für Bosnien als Auswanderungsland warb (vgl. Bethke 2018: 238). Der ländliche Zuzug deutscher Kolonisten nach Bosnien-Herzegowina beschränkte sich auf den eher flachen bis hügeligen Norden des Landes - die Posavina und die Semberija. Dort existierten bis zum Zweiten Weltkrieg und der organisierten Aussiedlung ins Dritte Reich deutsche Dörfer. Die Kolonisierung begann 1879 - nur ein Jahr nach der österreichisch-ungarischen Okkupation. Die Siedler kamen aus „Baden, dem Rheinland, Westfalen, Hannover und Oldenburg in die neu gegründete erste deutsche Siedlung in Bosnien mit dem Namen Windthorst, so benannt nach Bismarcks katholischem Gegenspieler Ludwig Windthorst in der Zeit des so genannten Kulturkampfes" (Scheuringer 2019: 36). Dem Dorf Windthorst, der auf rund 1.500 Bewohner anwuchs, folgten noch zwei größere deutsche ärarische Dörfer, Rudolfstal zwischen Banja Luka und Bosanska Gradiška und Franzjosefsfeld in der Nähe von Bijeljina, sowie 12 kleinere deutsche ärarische Dörfer. Dazu kamen deutsche Fabrikarbeitersiedlungen etwa in Zenica und Žepče sowie die Handwerkerviertel in Banja Luka und Bijeljina. Insgesamt dürfte die Anzahl dieser ,primär bäuerlichen deutschen Bevölkerung Bosniens zum Ende der österreichischen Zeit bei etwa 8.000 gelegen haben" (Scheuringer 2019: 38).

$\mathrm{Zu}$ dieser ländlichen Ansiedlung deutschsprachiger Kolonisten reiht sich auch ein Zuzug in die größeren Städte Bosnien-Herzegowinas. So betrug 1910 die Zahl der Personen mit Deutsch als Muttersprache im Kreis Sarajevo 6.495 Personen (2,26 \%). 
Damit war die deutsche Sprachgruppe nach der bosnischen/kroatischen/serbischen die zweitgrößte im Kreis, gefolgt von der sogenannten „spaniolischen“ (Ladino-)Sprachgruppe mit 5.441 Sprechern (1,89 \%) (vgl. Ergebnisse der Volkszählung 1912: 50). Bei der Zuwanderung in die Städte handelte es sich um „Unternehmer und Facharbeiter sowie Experten und vor allem um Beamte“ (Bethke 2018: 240).

Doch die Zahl der Deutschsprachigen in den Städten war viel größer. Das hängt damit zusammen, dass Deutsch die Lingua franca der Donaumonarchie war - auch in Bosnien-Herzegowina. Diesem Umstand trug der besondere völkerrechtliche Status dieses Okkupationsgebietes bzw. des späteren Reichslandes bei. Bosnien-Herzegowina war weder Teil der österreichischen noch der ungarischen Reichshälfte, sondern war direkt dem Gemeinsamen Finanzministerium Österreich-Ungarns unterstellt. Obwohl es zur Okkupationszeit keine festen Regelungen über den Amtssprachengebrauch in Bosnien-Herzegowina gab, etablierte sich Deutsch als Sprache des sogenannten inneren Amtsverkehrs, der höheren Militärränge sowie der Korrespondenz mit der Regierung in Wien - viele, besonders höhere Beamte waren zweisprachig. Darüber hinaus setzte sich Deutsch als gemeinsame Verkehrs- und Prestigesprache in den Kreisen der zugezogenen Beamten, Unternehmer und Experten durch, die aus unterschiedlichen Teilen der Monarchie stammten und in vielen Fällen treue Monarchiebürger waren (vgl. Memić 2019a: 189f.).

\section{PIONIERIN DER BOSNISCHEN ETHNOGRAFIE}

Eine dieser treuen Monarchiebürgerinnen im damaligen Bosnien-Herzegowina war auch Milena Preindlsberger-Mrazović. Ihr Engagement in einem Satz zu beschreiben, käme viel zu kurz. Wofür Preindlsberger-Mrazović heutzutage in Bosnien-Herzegowina am meisten bekannt ist, sind ihre journalistischen und ethnografischen Arbeiten. Die gebürtige Kroatin gilt als erste Journalistin und Chefredakteurin bzw. Herausgeberin in der Geschichte Bosnien-Herzegowinas. Da Preindlsberger-Mrazović jedoch hauptsächlich auf Deutsch schrieb, blieben ihre Werke dem bosnisch-herzegowinischen $\mathrm{Pu}-$ blikum jahrzehntelang relativ unbekannt. Erst nach dem letzten Krieg in Bosnien-Herzegowina und einem steigenden Interesse an der österreichisch-ungarischen Epoche wurden einige ihrer Arbeiten auch ins Bosnische übersetzt.

Preindlsberger-Mrazović entstammt einer typischen „kuferaši“-Familie. Geboren wurde sie in den 1860er Jahren. Einige Quellen sprechen von Wien als Geburtsort, andere wiederum von Bjelovar, vom einem „Dorf in Türkisch Kroatien“ oder sogar von Bosnien (s. Džambo 2016: 173f.). Ihre der Herkunft nach adelige Familie stammt aus dem kroatischen Bjelovar. Der Vater Andrija (Andreas) Mrazović wurde als hoher Beamter bereits 1878 nach Bosnien-Herzegowina entsandt. Dort bekleidete er das Amt eines Kreissekretärs in Banja Luka. Einige Jahre später folgten ihm seine Frau und Tochter Milena. Spätestens 1884/85 wissen wir, dass die Familie Mrazović von Banja Luka nach Sarajevo zog: In diesem Jahr unterrichtete Milena Mrazović an der dortigen Mädchenschule der Barmherzigen Schwestern ehrenamtlich (Džambo 2016: 176). In Sarajevo lebte Mrazović bis Anfang 1919, als sie das neu gegründete Jugoslawien verlassen musste und sich in Wien niederließ. 
In diesen gut 30 Jahren entstand ein einzigartiges journalistisches und ethnografisches Euvre, das erst rund 90 Jahre später in Bosnien-Herzegowina der breiteren Öffentlichkeit bekannt wurde. An dieser Stelle seien nur einige wichtige Stationen im Leben von Milena Preindlsberger-Mrazović aufgelistet: Als im Jahre 1884 die deutschsprachige Zeitung „Bosnische Post“ gegründet wurde, wurde sie dort als Lehrling aufgenommen. Doch sehr bald avancierte sie zu einer der besten Journalistinnen im Redaktionsteam. Nach nur fünf Jahren übernahm sie 1889 die Zeitung als Eigentümerin, Herausgeberin und Chefredakteurin.

\begin{abstract}
Als Bosnische Post 1894 ihr zehnjähriges Jubiläum feierte, unterstrich die gesamte Weltpresse die Tatsache, dass gerade in Bosnien-Herzegowina, wo Frauen zur damaligen Zeit keine öffentlichen Funktionen ausüben konnten, eine Frau als Vertreterin der freiesten Profession auftrat. (Šarić 2004) ${ }^{1}$
\end{abstract}

1896 heiratete sie Dr. Josef Preindlsberger, der zur damaligen Zeit als Primarius an der chirurgisch-okulistischen Abteilung des bosnisch-herzegowinischen Landesspitals tätig war (vgl. Džambo 2016: 176). Nach ihrer Heirat verkauft sie die Zeitung und fängt an, als Journalistin und Publizistin für deutschsprachige Zeitungen zu arbeiten. In der Donaumonarchie galt sie als große Bosnien-Kennerin (Džambo 2016: 177), ihre Berichte anlässlich wichtiger politischer Ereignisse wie die Annexion von BosnienHerzegowina im Jahre 1908 oder das Sarajevo-Attentat von 1914 fanden Beachtung in der österreichisch-ungarischen Presse.

Parallel zur journalistischen Arbeit trieb Preindlsberger-Mrazović ihre ethnografischen und anthropologischen Arbeiten über Bosnien-Herzegowina voran. Im Jahre 1893 wurde ihr Buch „Selam“ in Berlin veröffentlicht, das sich einigen Skizzen aus der bosnisch-muslimischen Welt widmete. Milena Preindlsberger-Mrazović sah sich als Vermittlerin der bosnischen Kultur in der deutschsprachigen Öffentlichkeit.

\begin{abstract}
Milena Mrazović machte die deutschsprachige Kulturöffentlichkeit mit der Kultur und den Naturschönheiten Bosniens bekannt. Aber sie hat über das Land, in dem sie lebte und welches sie liebte, keine Märchen geschrieben (sie sammelte bosnische Volksmärchen und veröffentlichte sie in einem Buch), sie schrieb auch nicht beliebig über Bosnien. Sie kannte es tatsächlich gut, weil sie das Land, meistens zu Pferde, kreuz und quer durchreiste und damit in die entlegensten Orte kam. Sie betrat reiche und arme Häuser, sprach mit allen, fragte über alles nach. So erfuhr und schrieb sie vieles nieder, was so in Vergessenheit geraten wäre. Sie sammelte und zeichnete Legenden, Märchen, Rätsel, Volksbräuche, Rezepte auf ... (Tomašević 2017)²
\end{abstract}

Auf ihren Reisen durch Bosnien-Herzegowina erwarb sie bosnische Frauentrachten: Nach dem Zweiten Weltkrieg kam diese Trachtensammlung in den Besitz des

1 Übersetzt von Nedad Memić.

2 Übersetzt von Nedad Memić. 
bosnisch-herzegowinischen Landesmuseums. Die Gründung dieses Landesmuseums unterstützte Mrazović selbst im Jahre 1888. Nur ein Jahr später wurde sie als erste Frau zum Mitglied der Anthropologischen Gesellschaft in Wien ernannt. Im Jahre 1900 veröffentlichte sie in Innsbruck das „Bosnische Skizzenbuch“, fünf Jahre später erschienen dort auch ihre „Bosnischen Volksmärchen“, die erst 2009 eine bosnische Übersetzung bekamen (vgl. Tomašević 2017).

Anfang 1919 zog Milena Preindlsberger-Mrazović nach Wien. Für sie war diese Stadt „die Fremde“, wie sie selbst behauptete (Džambo 2016: 178). Als überzeugte Österreicherin war für sie und ihre Familie kein Platz im neu gegründeten jugoslawischen Staat. Jedoch blieb sie auch in Wien mit Bosnien verbunden: Sie half bosnischen Studenten, die nach dem Ersten Weltkrieg nach Wien kamen. In ihrer Wohnung besaß sie ein Zimmer im bosnischen Stil, ,in dem sie sich - so sagt man - am liebsten aufhielt“ (Tomašević 2017). Im Mai 1926 hielt sie in der Wiener Urania ihre letzte öffentliche Lesung - auch diesmal wählte sie Bosnien als Thema. Ein halbes Jahr später starb Preindlsberger-Mrazović: Österreichische Zeitungen feierten sie in ihren Nachrufen als „berühmte bosnische Schriftstellerin“.

\section{MRAZOVIĆS BOSNIEN-BILD}

Bereits als Herausgeberin und Chefredakteurin der Tageszeitung „Bosnische Post“ zeigte sich Milena Preindlsberger-Mrazović regierungsnah. Die „Bosnische Post“ war zwar offiziell ein unabhängiges Blatt, propagierte aber schon von Anfang an eine monarchietreue Linie. Darauf deutet auch die Tatsache hin, dass das Blatt bei der Gründung sehr schnell eine Konzession von der Regierung bekam. Außerdem unterstützte das österreichisch-ungarische Außenministerium ab Oktober 1884 die Zeitung mit 100 Abonnements (vgl. Bethke 2018: 241). Es wurde zudem kein einziger Fall berichtet, in dem eine Ausgabe der „Bosnischen Post“ - immerhin existierte die Zeitung 35 Jahre lang, davon 22 Jahre als Tageszeitung - beschlagnahmt wurde, was „,vom ,Geist" dieser Zeitung Zeugnis gibt" (Džambo 2016: 185).

Obwohl kroatischen Ursprungs war Preindlsberger-Mrazović eine Vorzeigebürgerin der Doppelmonarchie - sie entstammte einer typischen k. u. k. Beamtenfamilie, bildete sich in Budapest aus und feierte ihre professionellen Erfolge in einem peripheren österreichisch-ungarischen Land, das damals als eine Art „Experimentierfeld in technologischer und sozialer Hinsicht" galt (vgl. Ruthner 2018: 41f.). Tatsächlich war Bosnien-Herzegowina das letzte Territorium, das ins Machtgefüge des Habsburgerreiches eingegliedert wurde. Während zu dieser Zeit in anderen Teilen dieses Vielvölkerstaates nationale Bewegungen auf mehr Selbstbestimmung und Unabhängigkeit pochten, konnte sich der eher konservative und komplexe k. u. k. Militär- und Verwaltungsapparat in Bosnien-Herzegowina als Stabilisierungs- und Modernisierungsfaktor vorerst behaupten. Österreich-Ungarn konnte einerseits eine Reihe bedeutender Infrastrukturprojekte in Bosnien-Herzegowina realisieren, andererseits bildete sich zu dieser Zeit auch eine Intelligenzschicht in den Städten, die sich von revolutionären Ideen in Europa inspirieren ließ. Die Donaumonarchie scheiterte aber in Bosnien-Herzegowina an der sozialen Frage: Die Agrarfrage wurde bis nach dem Ersten Weltkrieg 
nicht gelöst, das Schulsystem nach dem österreichischen Modell (z. B. mit Realgymnasien in Sarajevo, Mostar usw.) war zwar etabliert, die Zahl der Analphabeten war aber auch am Ende der k. u. k. Zeit extrem hoch. 1910 konnten 87,84 Prozent der Gesamtbevölkerung Bosnien-Herzegowinas weder lesen noch schreiben (Die Ergebnisse der Volkszählung 1912: 42).

Als letzte große Bahnstrecke der k. u. k. Zeit wurde die Bosnische Ostbahn am 4. Juli 1906 in Betrieb genommen. Der Bau dieser Bahnstrecke erfolgte aus militärisch-strategischen Gründen und war einer der herausforderndsten und teuersten im damaligen Bosnien-Herzegowina. Ein Baukilometer dieser Strecke kostete 450.000 Goldkronen. Der Streckenverlauf führte von Sarajevo zur osmanischen Grenze in Uvac und hatte auch eine Abzweigung nach Vardište an der bosnisch-serbischen Grenze. Die Länge der Ostbahnstrecke betrug 161,5 Kilometer. ${ }^{3}$ Da sie vor allem militärischen Zwecken diente (Österreich-Ungarn unterhielt Garnisonen in Goražde, Foča und Čajniče) und sich kaum wirtschaftlich rentierte, versucht man, die bosnische Ostbahn touristisch zu erschließen. Der Reiseführer Die Bosnische Ostbahn. Illustrierter Führer auf den bosnisch-hercegovinischen Staatsbahnlinien Sarajevo-Uvac und Megjegje-Vardište von Milena Preindlsberger-Mrazović wurde 1908 bei A. Hartleben's Verlag in Wien und Leipzig herausgegeben. Der Reiseführer ist umfangreich und zählt rund 190 Seiten, hat zwei Karten und mehrere Textzeichnungen aus dem bosnischen Leben und den bosnischen Landschaften der damaligen Zeit, die aus der Feder von Ewald Arndt und Ludwig Hans Fischer stammen. Die Autorin nutzte u. a. auch Fotos des tschechischen Fotografen František Topič, der eine Zeit lang im bosnisch-herzegowinischen Landesmuseum als Bibliothekar tätig war. Topič gilt als Pionier der Fotografie in Bosnien-Herzegowina.

Preindlsberger-Mrazović beginnt ihren Reiseführer in Sarajevo und folgt anschlieBend dem Streckenverlauf der Bosnischen Ostbahn bis an die osmanische und serbische Grenze. Bereits bei der Beschreibung der bosnisch-herzegowinischen Landeshauptstadt preist die Autorin die Ostbahn als ,elementare, treibende Macht, die der moderne kulturelle Wachstumsprozess darstellt“ (Mrazović 1908: 5). Die Bosnische Ostbahn ist für sie ein Werkzeug im Dienste der österreichisch-ungarischen Modernisierung des Landes.

\begin{abstract}
Schon vor der Abfahrt in Sarajevo zählt sie auf, was alles die Regierung bis dahin für die Modernisierung der Landeshauptstadt geleistet hatte: Flussregulierung, Fabrikanlagen, Landesspital, Schlachthaus, Aktienbrauerei, Holzlager u. a. [...] Das sie faszinierende Moderne sah sie aber noch viel mehr in der Bahnstrecke mit all den dazugehörigen Bauten und Einrichtungen. (Džambo 2016: 195)
\end{abstract}

Preindlsberger-Mrazović präsentiert sich in der „Bosnischen Ostbahn“ als ausgesprochene Kennerin des Landes und seiner Bevölkerung. Ihre Beschreibungen der Orte und Landschaften entlang der Strecke sind umfassend und auffallend detailreich: sowohl geografisch und historisch als auch kulturell und sogar geologisch. Als jemand, der jahrelang bosnische Märchen und Volkslegenden sammelte, flicht sie diese

3 Vgl. www.zfbh.ba (31.01.2020). 
geschickt in die Reisebeschreibung hinein, sodass sich dieser Reiseführer wie ein gut recherchiertes ethnografisches Werk liest. Die Faszination der Autorin vom Modernen, an das sich die einheimische Bevölkerung erst gewöhnen müsse, verfolgt die Leserinnen und Leser des Reiseführers bis zum Ende:

Eine große moderne Bahn, die an einem Fußsteig endet! Schneidender kann sich der Gegensatz zwischen Orient und Okzident auf dem Balkan nicht ausdrücken. Ein Gegensatz, in dem die Gewähr für eine bessere Zukunft liegt. Bis nun kam der Fremde immer nur als Zerstörer hierher; jetzt zum ersten Male kommt er als Gebender. Anderwärts mag die Herstellung einer neuen Eisenbahnlinie zu den gleichgiltigen, geschäftlichen Ereignissen gerechnet werden; hier in Ostbosnien ist sie eine Tat der ausgleichenden Gerechtigkeit in der Geschichte der Menschen. Es darf uns mit Befriedigung erfüllen, daß dieses Werk von ÖsterreichUngarn ausgegangen ist, und es wird seine Krönung erst dann erfahren, wenn es von Vardište aus heißen wird: „Vorwärts!“ (BOB: 170f.)

Kaum ein anderes Zitat aus Preindlsberger-Mrazovićs Texten wie dieses illustriert den festen Glauben der Autorin an die zivilisatorische Rolle, die Österreich-Ungarn in Bosnien-Herzegowina zuteilwurde. „Mrazović identifizierte sich vollends mit dem kulturellen und zivilisatorischen Vorhaben Österreich-Ungarns in Bosnien-Herzgowina“ (Džambo 2016: 196). Gleichzeitig zeigt sie auch Verständnis für die traditionelle Lebensweise der Bosnier: „An vielen Stellen bedauerte sie das Verschwinden eben eines solchen Bosnien infolge der unaufhaltsamen Modernisierungsprozesse“ (Džambo 2016: 196). In diesem Widerspruch reflektiert sich das Bosnien-Bild von Milena Preindlsberger-Mrazović: Ihre Beschreibungen der bosnischen Geschichte, Bräuche und Traditionen tragen durchaus romantisierende Züge, gleichzeitig ist sie von den Vorteilen der modernen Zeit, deren Symbol die moderne Bahn ist, fest überzeugt.

\section{ZUM BEGRIFF DER KULTURSPEZIFIKA}

Ein Kulturspezifikum ist „ein Element, das einem bestimmten kulturellen System eigen ist, und in anderen nicht erscheint" (Ndeffo Tene 2004: 187). In der Übersetzungswissenschaft hat sich für kulturspezifische Begriffe der Terminus „Realie“ etabliert. Laut Markstein kann man die Realia auf zwei Ebenen definieren: Zum einen sind sie „Elemente des Alltags, der Geschichte, der Kultur, der Politik u. dgl. eines bestimmten Volkes, Landes, Ortes, die keine Entsprechung bei anderen Völkern, in anderen Ländern, an anderen Orten haben“. Zum anderen spricht Markstein von den Realia als „Identitätsträgern eines nationalen/ethnischen Gebildes, einer nationalen/ethnischen Kultur“, die „einem Land, einer Region oder einem Erdteil zugeordnet“" werden (Markstein 1998: 288).

Markstein definiert vier translatorische Lösungen für die Übertragung von Realia aus der Ausgangs- in die Zielsprache (vgl. Markstein 1998: 290f.): 1) Zitatwort, wenn der Ausdruck unverändert in die Zielsprache übernommen wird; 2) Lehnübersetzung; 3) Analogiebildung, wenn ein sinngemäß entsprechendes Wort der Zielsprache verwendet wird, sowie 4) die kommentierende Übersetzung. Diese Klassifizierung dient 
uns als Basis, um die vorhandenen Kulturspezifika in der „Bosnischen Ostbahn“ einer Analyse zu unterziehen.

Obwohl die Bosnische Ostbahn keine Übersetzung ist, sondern ein Werk in deutscher Sprache, war es für seine Autorin eine Herausforderung, zahlreiche Begriffe aus der bosnischen Kultur, Geschichte und Geografie ins Deutsche zu übertragen. Die Zielgruppe der „Bosnischen Post“ waren in erster Linie Touristen aus der Monarchie, die nach Bosnien-Herzegowina kamen, sowie deutschsprachige Bevölkerung, die damals im Lande lebte. Die Aufgabe dieses Reiseführers war, ihnen die bosnische Lebensweise und die Landschaften entlang der Strecke attraktiv zu machen und sie zu einer Fahrt $\mathrm{zu}$ animieren.

\section{KULTURSPEZIFIKA IN DER „BOSNISCHEN OSTBAHN“}

Die Autorin der „Bosnischen Ostbahn“ musste sich bei ihrem Vorhaben, bosnische Kulturspezifika ins Deutsche zu übertragen, vor etliche Herausforderungen stellen. Sie betrafen mehrere Sprachebenen: von der Orthografie, über Morphologie und Lexikologie bis hin zur Idiomatik.

\subsection{Orthografie}

Bei der Schreibung der bosnischen Toponyme sowie Vor- und Familiennamen bleibt die Autorin konsequent - sie werden in der damals geltenden bosnischen bzw. serbokroatischen Rechtschreibung ins Deutsche übertragen. Um den Leserinnen und Lesern die Aussprache zu erleichtern, fügte die Autorin am Anfang eine Anleitung zur Aussprache hinzu.

Eine gewisse Anpassung ans Deutsche findet man in der Schreibung der Komposita. So werden manchmal bosnische Realien ins Deutsche als Zitatwörter übertragen, die Autorin verwendet aber den Bindestrich und adaptiert diese eigentlich an die deutsche Rechtschreibung, z. B. im Falle von Toponymen wie Crna-rijeka, Kozija-Ćuprija, Kruševićka-pećina, Markova-kula, Paljanska-Miljacka, Ravna-planina usw. oder etwa bei religiösen Festen wie Mala- und Velika-Gospojina oder Sveti-Jovo usw. Die gebundene Schreibweise finden wir auch im Falle von sogenannten Hybrid-Komposita, bei denen das Grundwort deutsch und das Bestimmungswort bosnisch ist, z. B.: Borija-Kuppen, Bistrica-Bach, Jahorina-Tunnel, Kiseljak-Quelle, Nizam-Posten, VolujakGipfel usw.

Bei der Groß- und Kleinschreibung schwankt die Autorin zwischen der bosnischen und der deutschen Schreibweise: Kozija-Ćuprija, aber Kruševićka-pećina.

\subsection{Morphologie}

Im Bereich der Flexion adaptiert Mrazović bosnische Realia an das grammatische System des Deutschen: Dies ist vor allem am Genus der übertragenen Toponyme sichtbar. Jedoch schlägt sie bei der Adaption unterschiedliche Wege ein. Einerseits behält sie bei vielen Toponymen das Genus der Ausgangssprache - was der deutschen Sprachpraxis durchaus entspricht: 
„Links im Abgrunde die Miljacka.“ (BOB: 15)

„Die Mitte der Romanija aber nimmt ein wie ins Ungemessene gehendes Weideland ein, der Glasinac [...]" (BOB: 25)

So denkt man sich den Zlatibor (= Goldföhre) als die Urheimat des Bäumchens, das sich goldene Nadeln gewünscht. (BOB: 168)

Das Genus wird auch dann beibehalten, wenn ein Kompositum ins Deutsche übertragen wird, obwohl die appellativische Konstituente des Kompositums gar keine Realie ist:

[...] die zweifellos das Kalkplateau der Gosinje-planina, eines Romanija-Astes, darstellt. (BOB: 47)

[...] an dem für das Volk eine vage Reminiszenz an die „Prača Biskupija“, das „Bistum Prača“, hängt. (BOB: 44)

Sein äußerster Talast reicht, wie wir wissen, bis an die Kovač-Planina südlich von Čajniče hinan [...] (BOB: 95)

In diesen Fällen hätte man die bosnischen Appellativa planina/Planina und Biskupija durchaus ins Deutsche übersetzen können, für die Autorin waren sie aber feste Bestandteile der Eigennamen, die sie samt ihrem Artikel aus der Ausgangssprache ins Deutsche übertrug.

Ähnlich geht sie auch bei der Übernahme anderer bosnischer Appellativa ins Deutsche vor: In der Regel wird das Genus aus der Ausgangssprache mitübernommen:

Würdevoll kehrt er dann durch die Čaršija (Marktviertel) heim. (BOB: 67)

Um eine feste, mit Schießscharten bewehrte Kula (Turm), in die man sich bei einem Angriffe zurückzog [...] (BOB: 78)

Hat man einmal die Karaula mit dem Halbmond und Stern, hinter sich, so ist man schnell in Priboj [...] (BOB: 129f.)

Auf halbem Wege, bei der Mündung des Bistrica-Baches, trifft man nächst einem türkischen Weiler auf einen Han (türkische Herberge). (BOB: 132)

Unweit davon erhebt sich an einer Straßenecke das Turbe (Grabmal) der Schwester Mehmed Paschas [...] (BOB: 147)

Ein anderer Aspekt der Flexion ist die Pluralbildung bei einigen übernommenen Realia. 
[...] deren naive Bauart auf einheimische Dungjers (Meister, die jedes Bauhandwerk ausüben) hinweist. (BOB: 83f.)

Hier wurde das bosnische Lexem dunđer (ein Maskulinum in der Bedeutung , Meister $\left.{ }^{6}\right)$ ins Deutsche übernommen und mit dem grammatischen Morphem -s für die Pluralbildung versehen. Den $s$-Plural finden wir auch bei einigen Feminina, hier mit einem Apostroph:

Dieser entspringt dem oberen Rande der Waldwiesen von Pale und dient für die großen Hajka's, die Treibjagden, als Zusammenkunftsort.

Manchmal bedient sich die Autorin der Analogie: So überträgt sie das bosnische Wort vila (,Fee') und führt es ins System des Deutschen ein, indem sie es als ein deutsches Femininum behandelt und das grammatische Morphem -en für die Pluralbildung verwendet:

Er scheute niemand außer Gott den Einen, da doch die nationalen Vilen (Feen) ihn beschützten. (BOB: 28)

\subsection{Translatorische Lösungen}

Wenn wir der Klassifizierung von Markstein folgen (s. Kapitel 5), entscheidet sich die Autorin der „Bosnischen Ostbahn“ in den meisten Fällen für kommentierte Übersetzungen bzw. Übernahmen. Das ist angesichts der Aufgabe, die sich Milena PreindlsbergerMrazović beim Verfassen dieses Werkes stellte, sowie des eher geringen Bekanntheitsgrades von Bosnien-Herzegowina als Reisedestination zu Beginn des 20. Jahrhunderts durchaus verständlich. Aus dem Buch ist ihr Bestreben, bosnische Kulturspezifika egal ob sie als Appellativa oder als Toponyme vorkommen - möglichst detailliert und konsequent zu erklären, klar herauszulesen.

Die häufigste Methode, die sie dabei wählt, ist die Übernahme des Begriffes aus der Ausgangssprache, der in den meisten Fällen ein Kommentar (Übersetzung) in deutscher Sprache in Klammern oder als Apposition folgt - manchmal mit der Verwendung von Anführungszeichen. Einige Beispiele dieser Methode sind: Careve Vode (Kaiserquell) - Kozija-Ćuprija (Ziegen-Brücke) -,,Starigrad“ (Alte Burg) -,,Han Pale“ (Han = Herberge) - Orlovina-stijena (Adlergefels, 1629 m); - Jerin-put (Weg der Fürstin Irene) - Jeringrad (Irenenburg) - Ravna-planina, das „Ebene Gebirge“ - Topski-put (Kanonenweg) - Strmica (strmo = steil) - die Stadt der "Teferić“ (sic!), der in einem feisten Lämmchen am Spieße gipfelnden sommerlichen Ausflüge usw.

Seltener wird die umgekehrte Methode verwendet: Zuerst wird die deutsche Übersetzung angeführt und dann durch die bosnische Entsprechung kommentiert: Aussichtswarte des Hodža (Hodžina-ravan) - Lehen (Timar) - [der] Pflastersteig, die „Kaldrma" - das Haus für die Gaste (Musafirhana) - die vom oberen Plateau-Rande leicht zugängliche Höhle, die „Novakova-pećina“ usw. 
Ebenfalls selten werden Zitatwörter verwendet, wie der folgende Satz zeigt:

Auf seinen nackten Kalkgipfel ist der serbische Nationalheros Marko Kraljević im Galopp hinaufgeritten und ganz oben sieht man noch den Hufabdruck des braven ,Šarac“ [...] (BOB: 36)

In diesem Fall hätte das Substantiv Šarac (,Schecke') zumindest kommentiert werden sollen, auch beim Substantiv Kraljević handelt es sich um keinen Familiennamen, sondern um ein appellativisches Nomen (,Königssohn'), der zum festen Namensteil dieses epischen Helden wurde. Im späteren Verlauf des Werks spricht die Autorin jedoch von Marko, dem Königssohne (BOB: 142). Zitatwörter finden wir auch bei der Beschreibung von Religionsbüchern, die im Kloster Sopotnica bei Goražde verfasst wurden:

\begin{abstract}
Hilarion Ruvarac zählt sie auf: „Psaltir“, herausgegeben von dem Greise Božidar Goraždanin, mit Hilfe des Jeromonach Theodor, im Jahre 7029 oder 1529; „Liturgija“, vom demselben, gedruckt im Jahre 7027 oder 1527, und „Molitavnik“ von demselben und Djakon Radoje im Jahre 7032 oder 1532. (BOB: 79f.)
\end{abstract}

Bei mehrfachen Nennungen eines Kulturspezifikums bzw. einer Realie wendet die Autorin das Prinzip an, dass die Erstnennung in Form einer kommentierten Übersetzung und weitere Nennungen dann als Zitatwörter erscheinen. Dafür dient uns das Beispiel der Realie Karaula. Zum ersten Mal wird sie als kommentierte Übersetzung auf S. 122 erwähnt: Karaula (Grenzwachhaus), danach wird das Wort neun Mal unkommentiert verwendet, an einer Stelle sogar in der Pluralform mit Apostroph Karaula's (BOB: 150).

\title{
6.4 Idiomatik
}

Um die bosnische Lebensweise und Traditionen dem deutschsprachigen Publikum näherzubringen, scheut Preindlsberger-Mrazović auch nicht davor zurück, bosnische Idiome oder Redewendungen ins Deutsche zu übersetzen. Als Zweisprachige macht sie es dort, wo der Sinn der idiomatischen und anderen Wendungen auch für die deutschsprachige Leserschaft leicht verständlich ist. In einigen Fällen führt sie ein Idiom bzw. eine Redewendung zuerst in der Ausgangssprache an und überträgt sie dann in Form eines Kommentars ins Deutsche:

Sobald der Tunnel uns entläßt, finden wir uns plötzlich ,hinter dem Rücken Gottes ", wie der Bosnier sagt. (BOB: 15)

„Die Zeit baut auf und die Zeit reißt nieder (vrijeme gradi i vrijeme razgradi)", meint der Bosnier resigniert [...] (BOB: 80)

Dies ist der Ort, „wo“, um sich mit den Dörflern von Strmica auszudrücken, ,einem die Welt offen steht". (BOB: 109) usw. 


\title{
6.5 Legenden
}

In ihrer Beschreibung des Landes und der Leute entlang der bosnischen Ostbahnstrecke zieht die Autorin u. a. auch lokale Legenden heran. Die zwei Legenden, die in Ostbosnien verbreitet waren und wiederholt im Werk vorkommen, sind die Sagen von Kraljević Marko und Prokleta Jerina (die verdammte Irene). ${ }^{4}$ Beide Legenden entstammen dem serbischen Kulturkreis, waren aber auch seit Jahrhunderten bei anderen südslawischen Völkern verbreitet. Milena Preindlsberger-Mrazović nimmt sie in ihren Reiseführer auf als Beitrag zur ethnografischen Erforschung des damaligen Bosnien. In der „Bosnischen Ostbahn“ dienen sie als Stilmittel dazu, Bosnien als ein traditionelles, sagenumwobenes Land zu präsentieren, das in seiner Ursprünglichkeit noch entdeckt werden muss. Durch diese Legenden schafft die Autorin einen Gegenpol zur modernen Bahn, die im Zeichen des technologischen und zivilisatorischen Fortschritts steht. Zur Illustration seien hier zwei Textpassagen angeführt:

\begin{abstract}
$\mathrm{Da}$ aber so vollständig vergessen ist, wer sie gebaut und welche Schicksale sie gehabt, ging offenbar wider das Empfinden des Volkes, und so ließ es hier den Heros der Südslaven, Marko Kraljević, gefangen sein und bezeichnet deshalb die Ruine auch als Markova-kula (Schloß des Marko). (BOB: 141)
\end{abstract}

Durch einen bedeutenden Umweg kann man das Drina-Ufer schon bei dem bereits erwähnten Hrtar gewinnen, wo ein aus dem Strome aufsteigender Fels die Ruine einer Jerina-Burg trägt. Die Sage läßt das für den Bau der Burg nötige Materiale durch Ziegen auf den Fels hinauftragen und den Mörtel mit Eiweiß anrühren. Dadurch erklärt man sich die Möglichkeit der Bauausführung und die Härte der Mauerreste. Der der Drina zugewendete Felshang zeigt eine Höhle, in der die grausame Burgherrin ihre Gefangenen verhungern ließ. (BOB: 153f.)

\subsection{Exkurs: Demonyme}

Es wurde bereits betont, dass Milena Preindlsberger-Mrazović in Österreich-Ungarn als Kennerin Bosnien-Herzegowinas galt. Seit dem Mittelalter gilt dieses Balkanland als ein Schmelztiegel unterschiedlicher Religionen und Ethnien. In der „Bosnischen Ostbahn" reist die Autorin in Gegenden von Bosnien-Herzegowina, die vorwiegend von serbisch-orthodoxen Christen und Muslimen bewohnt sind. Um auf orthodoxe Christen ethnisch zu referieren, verwendet die Autorin auch die ethnische Bezeichnung Serben, so z. B. in der Beschreibung der Stadt Foča:

Hierin macht sich besonders die von der Čaršija ziemlich abschüssig zur Drina niedergehende Stadtteil bemerkbar, der den etwa ein Drittel der Bewohner

4 Beiden Sagengestalten liegen historische Persönlichkeiten zugrunde: Zur Sagengestalt von Kraljević Marko (der serbische König Marko Mrnjavčević) s. Calic (2016: 36). Im Falle der Legende von Prokleta Jerina handelt es sich um die griechische Prinzessin Irene Kantakouzene, die die Ehefrau des serbischen Despoten Đurađ Branković (15. Jahrhundert) war. 
ausmachenden Serben gehört, und in dem sich auch ihre Kirche und ihre konfessionelle Elementarschule hinter Mauern versteckt.

Die bosnischen Muslime nennt sie meistens Moslims, auf ihre Religion bzw. Religions-zugehörigkeit referiert sie adjektivisch als islamitisch:

Nun dringt das vorwiegend von Moslims bewohnte Bergviertel „Sobunar“ über das Geleise hinaus und bis zu den Felswänden vor. (BOB: 10)

Reiche Quellen netzen den der erodierenden Wirkung des Wassers noch kraftvoll widerstehenden Boden, der, durchwegs ein Privatbesitz islamitischer Großgrundherren, ungezählten Viehherden eine ausgezeichnete Weide bietet. (BOB: 29)

Die Bewohner Bosniens nennt Preindlsberger-Mrazović meistens Bosnier, seltener Bosniaken (zwölf zu vier Nennungen). Im Werk beziehen sich beide Begriffe auf alle Bewohner Bosniens:

Schwerer begehbar als diese Ecke der Romanija ist wohl keines der vielen unwirtlichen Gebiete des Landes, da sie selbst die außergewöhnliche Orientierungsgabe des Bosniers narrt. (BOB: 23)

Nur Eines vertragen die vom Lim nicht: mit zu den Bosniaken vom Podrinje gerechnet zu werden, denen sie nachsagen, sie hatten Nadeln gesaet, um Eisenstangen zu ernten [...] (BOB: 112)

Auf die Bewohner der Herzegowina wird in der „Bosnischen Ostbahn“ nur einmal referiert, und zwar als Hercegovcen:

Mit den Hercegovcen, also jenen von der linken Lim-Seite, vertragen sich die Onostranci sehr gut; mit den Bosniaken wollen sie jedoch nicht viel zu tun haben. (BOB: 161)

\section{SCHLUSSFOLGERUNG}

Milena Preindlsberger-Mrazović demonstriert in der „Bosnischen Ostbahn“ ihre Faszination von Bosnien und seinen Leuten durchaus eindrucksvoll: Sie ist eine großartige Kennerin eines Landes, das am Ende des 19. und am Anfang des 20. Jahrhunderts seinen Anschluss an mitteleuropäische Kulturströmungen sucht. Die Autorin versteht sich dabei als eine eifrige Vermittlerin zwischen zwei Kulturen, ihr Bestreben ist, dem deutschsprachigen Publikum Bosnien-Herzegowina so detailliert wie möglich darzustellen - die Bosnische Ostbahn ist demnach nicht nur ein Reiseführer, es liest sich zugleich wie ein Roman, aber auch wie eine ethnografische, historische und geologische Studie. Darin versucht die Autorin, möglichst viele bosnienspezifische Begriffe möglichst konsequent zu erklären - daher ist ihre Entscheidung, die kommentierte 
Übersetzung bzw. Übernahme als häufigste Lösung bei der Übertragung bosnischer Kulturspezifika ins Deutsche zu verwenden, logisch. Die Bosnische Ostbahn hat ihre Aufgabe als Reiseführer bei Weitem übertroffen - dieses Werk bleibt uns als lebende Erinnerung an eine außerordentliche und mutige Frau, deren literarisches Erbe auch ein Jahrhundert lang später für Inspiration sorgt.

\section{Literatur}

\section{Primärliteratur}

BOB = PREINDLSBERGER-MRAZOVIĆ, Milena (1908) Die Bosnische Ostbahn. Illustrierter Führer auf den bosnisch-hercegovinischen Staatsbahnlinien SarajevoUvac und Megjegje-Vardište. Wien/Leipzig: A. Hartleben's Verlag.

\section{Sekundärliteratur}

BETHKE, Carl (2018) „Einwanderung und Kolonisten im k.u.k. Bosnien-Herzegowina. Überblick mit ,bosniakischen Perspektiven““. In: C. Ruthner/T. Scheer (Hrsg.), 237-250.

CALIC, Marie-Janine (2016) Südosteuropa. Weltgeschichte einer Region. München: C.H. Beck.

Die Ergebnisse der Volkszählung in Bosnien und der Hercegovina vom 10. Oktober 1910 (1912). Zusammengestellt vom Statistischen Departement der Landesregierung. Sarajevo: Landesregierung für Bosnien und die Hercegovina.

DŽAMBO, Jozo (2016) „Milena Preindlsberger-Mrazović - eine Publizistin zwischen Folklore und Modernität." In: V.Tutavac/I. Korotin (Hrsg.), Wir wollen der Gerechtigkeit und Menschenliebe dienen... Frauenbildung und Emanzipation in der Habsburger Monarchie - der südslawische Raum und seine Wechselwirkung mit Wien, Prag und Budapest. Wien: Praesens, 173-213.

KREVS BIRK, Uršula (2019) ,Zu einigen Aspekten des Deutschen als Kontaktsprache des Slowenischen." Linguistica 59/1, 155-173.

MARKSTEIN, Elisabeth (1998) „Realia.“ In: M. Snell-Hornby/H. G. Honig/P. Kußmaul/P. A. Schmitt (Hrsg.), Handbuch Translation. Tübingen: Stauffenberg.

MEMIĆ, Nedad (2019a) „Die Stellung des deutschen Transfergutes in der bosnischen Gegenwartssprache.“ In: S. Mešić/H. Scheuringer (Hrsg.), 61-78.

MEMIĆ, Nedad (2019b) „Besonderheiten der deutschen Verwaltungssprache im k. u. k. Bosnien-Herzegowina." Linguistica 59/1, 187-195.

MEŠIĆ, Sanela/Hermann SCHEURINGER (Hrsg.) (2019) Deutsch in Bosnien-Herzegowina. Germanistenkonferenz Sarajevo, 22.-24. März 2018. Sarajevo: Philosophische Fakultät der Universität Sarajevo, 30-40.

NDEFFO TENE, Alexandre (2004) (Bi)kulturelle Texte und ihre Übersetzung: Romane afrikanischer Schriftsteller in französischer Sprache und die Problematik ihrer Übersetzung ins Deutsche. Würzburg: Königshausen \& Neumann.

RUTHNER, Clemens (2018) „Bosnien-Herzegowina als k.u.k. Kolonie. Eine Einführung.“ In: C. Ruthner/T. Scheer (Hrsg.), 15-44. 
RUTHNER, Clemens/Tamara SCHEER (Hrsg.) (2018) Bosnien-Herzegowina und Österreich-Ungarn, 1878-1918, Annäherungen an eine Kolonie. Tübingen: Narr Francke Attempto.

SCHEURINGER, Hermann (2019) „Bosniens rudimentärster Kontakt mit dem Deutschen - Die Posavina als Nahhut der großen Schwabensiedlung." In: S. Mešić/H. Scheuringer (Hrsg.), 30-40.

ŠARIĆ, Salko (2004) „Dvije zaboravljene gospođe. Milena Preindlsberger-Mrazović i Jelica Belović Bernadzikowska, Austrijska književnost. “Most-časopis za obrazovanje, nauku i kulturu, Jg. 173/84, 28-31.

\section{Internetquellen}

Historija željeznica u Bosni i Hercegovini. www.zfbh.ba (30.01.2020).

TOMAŠEVIĆ, Dragana (2017) Milena Mrazović-Preindlsberger: prva novinarka u BiH. www.stav.ba (30.01.2020).

\section{Zusammenfassung \\ DEUTSCH-BOSNISCHER SPRACHKONTAKT AM BEISPIEL BOSNISCHER KULTURSPEZIFIKA IN MILENA PREINDLSBERGER-MRAZOVIĆS DIE BOSNISCHE OSTBAHN}

Österreich-Ungarns Rolle als Okkupations- und später auch Annexionsmacht in Bosnien-Herzegowina war u. a. auch von einem Versuch geprägt, das Land kulturell „,zu missionieren" und es an mitteleuropäische Kulturströmungen anzuschließen. Die beginnende Industrialisierung oder große Infrastrukturprojekte wie Bahnstrecken wurden dabei als notwendige Schritte betrachtet, um das Land „zu zivilisieren“. Die kroatischstämmige Journalistin, Ethnografin und Schriftstellerin Milena PreindlsbergerMrazović zählte zu den überzeugten MonarchiebürgerInnen in Bosnien-Herzegowina, die die k. u. k. Präsenz in diesem Land als Zivilisierungs- und Emanzipierungsmission verstand. Vor diesem Hintergrund entstand ihr Reiseführer Die Bosnische Ostbahn im Jahr 1908, in dem sie versuchte, das unbekannte Land entlang der bosnischen Ostbahnstrecke dem interessierten deutschsprachigen Reisepublikum näherzubringen. In diesem Beitrag konzentrieren wir uns auf Lösungen und Methoden, die die Autorin anwendete, um bosnische Kulturspezifika bzw. Realia ins Deutsche zu übertragen und sie auf diese Weise der bosnienunkudigen Leserschaft im deutschsprachigen Raum sowie zahlreichen k. u. k. Zuwanderern in Bosnien-Herzegowina zu präsentieren.

Schlüsselwörter: Bosnien-Herzegowina, Österreich-Ungarn, Sprachkontakt, Übersetzung, Kulturspezifika 


\section{Abstract \\ GERMAN AND BOSNIAN LANGUAGE CONTACT. BOSNIAN CULTURAL SPECIFICS IN MILENA PREINDLSBERGER-MRAZOVIĆ'S}

DIE BOSNISCHE OSTBAHN

After annexing Bosnia-Herzegovina, the role of Austria-Hungary in the territory was also shaped by an effort to culturally "missionize" the country and bring it closer to Central European cultural trends. The ongoing industrialization and huge infrastructure projects like the construction of railway lines were considered necessary steps in "civilizing" the province. The Croatian-born journalist, ethnographer and writer Milena PreindlsbergerMrazović was a loyal citizen of the Monarchy in Bosnia-Herzegovina, who understood the Austro-Hungarian presence there as a "civilizing and emancipating" mission. Her 1908 guidebook Die Bosnische Ostbahn ("The Bosnian Eastern Railway Line") was written with an effort to present the unknown land along the Bosnian Eastern Railway Line to German-speaking audiences. The paper analyzes the methods used by the guidebook author in transferring Bosnian culturally specific items to German with the aim of bringing them closer to the German-speaking readership abroad as well as to the numerous Austro-Hungarian immigrants in Bosnia-Herzegovina itself.

Keywords: Bosnia-Herzegovina, Austria-Hungary, language contact, translation, culturally specific items

Povzetek

NEMŠKO-BOSANSKI JEZIKOVNI STIK OB ZGLEDU BOSANSKIH KULTURNIH POSEBNOSTI V DELU DIE BOSNISCHE OSTBAHN MILENE PREINDLSBERGER-MRAZOVIĆ

Avstro-Ogrska je poleg okupacije in nato aneksije Bosne in Hercegovine imela med drugim namen, da bi deželo prosvetlila tudi v kulturnem smislu ter jo vključila v srednjeevropske kulturne tokove. Uvajanje industrializacije ali pa veliki infrastrukturni projekti kot denimo železniške proge so se pri tem zdeli nujni koraki, s katerimi bi deželo „,civilizirali". Novinarka hrvaškega rodu, etnografinja in pisateljica Milena Preindlsberger-Mrazović je bila ena izmed zavednih državljank in državljanov avstro-ogrske monarhije v Bosni in Hercegovini, ki je c.-kr. prisotnost v tej deželi razumela kot civilizacijsko ter emancipatorno poslanstvo. S tega gledišča je leta 1908 nastal njen vodič Die Bosnische Ostbahn (slov. Bosanska vzhodna železnica), s katerim je želela neznano deželo vzdolž bosanske vzhodne železniške proge približati za potovanja zainteresirani nemško govoreči publiki. Prispevek se osredinja na rešitve in metode, ki jih je v delu uporabila avtorica, da bi v nemščino prenesla bosanske kulturne posebnosti oz. realije in jih predstavila nemško govorečemu, o deželi še nepoučenemu bralstvu iz nemškega jezikovnega prostora ter tudi številnim c.-kr. priseljencem v Bosni in Hercegovini.

Ključne besede: Bosna in Hercegovina, Avstro-Ogrska, jezikovni stik, prevod, kulturne specifike 